\title{
Efeito Residual de Isoxaflutole após Diferentes Períodos de SECA ${ }^{1}$
}

\author{
Residual Effect of Isoxaflutole Under Different Dry Periods
}

\author{
MARCHIORI JR., O..$^{*}$, CONSTANTIN, J..$^{*}$, OLIVEIRA JR., R.S..$^{3^{*}}$, INOUE, M.H. ${ }^{4}$, \\ PIVETTA, J.P. ${ }^{5}$ e CAVALIERI, S.D. $6^{*}$
}

\begin{abstract}
RESUMO - O presente trabalho objetivou estudar o efeito residual de diferentes doses de isoxaflutole sob diferentes condições de períodos de seca, após a aplicação do herbicida, em dois solos de textura contrastante. Para isso, foram realizados dois ensaios simultâneos: no primeiro foram utilizadas doses de 0, 230 e $270 \mathrm{~g} \mathrm{ha}^{-1}$ de isoxaflutole em amostras de Latossolo Vermelho distroférrico nitossólico (textura argilosa); e, no segundo, as doses foram de 0, 180 e $200 \mathrm{~g} \mathrm{ha}^{-1}$ em amostras de Latossolo Vermelho distrófico (textura francoargilo-arenosa). Em cada ensaio, foram combinadas em esquema fatorial três doses, duas espécies bioindicadoras (Brachiaria decumbens e Panicum maximum) e sete períodos de seca após as aplicações do herbicida (0, 20, 40, 60, 80, 100 e 120 dias), com quatro repetições. As avaliações de controle foram feitas aos 15, 30, 45 e 60 dias após a semeadura dos bioindicadores. O isoxaflutole apresentou alta estabilidade ( $\geq 97 \%$ de controle) no Latossolo Vermelho distroférrico nitossólico, independentemente da dose, do bioindicador e do período de seca avaliados. A estabilidade foi menor no Latossolo Vermelho distrófico, porém o efeito residual ( $\geq 80 \%$ de controle) persistiu entre 25 e 50 dias e entre 50 e $>120$ dias, respectivamente, para $B$. decumbens e $P$. maximum, conforme dose (180 e $200 \mathrm{~g} \mathrm{ha}^{-1}$ ) e dias após a semeadura analisados. Esse fato evidenciou a maior sensibilidade de $P$. maximum ao isoxaflutole do que $B$. decumbens, sendo a atividade residual deste herbicida maior no solo de textura argilosa.
\end{abstract}

Palavras-chave: bioindicador, condições climáticas, persistência.

\begin{abstract}
This research was carried out to evaluate the residual activity of isoxaflutole applied under different conditions of simulated drought after herbicide application, in two soils of contrasting textures. Two experiments were simultaneously performed; in the first one rates of 0,230 and $270 \mathrm{~g} \mathrm{ha}^{-1}$ of isoxaflutole were applied to a heavy clay soil. In the second one, rates of 0,180 and $200 \mathrm{~g} \mathrm{ha}^{-1}$ were applied to a sandy clay loam soil. In both experiments, a $3 \times 2 \times 7$ factorial combination (three rates, two bioindicators - Brachiaria decumbens and Panicum maximum, and seven drought periods after herbicide application -0,20, 40, 60, 100 and 120 days) was set, with four replicates. Visual control of bioindicators was evaluated at 15, 30, 45 and 60 days after sowing. Isoxaflutole was very stable on the clay soil (control $\geq 97 \%$ ), despite the rates applied and bioindicator or drought periods evaluated. Herbicide stability was shorter at sandy clay loam soil, but consistent residual activity (control $\geq 80 \%$ ) was persistent up to 50 days for $\boldsymbol{B}$. decumbens and $>120$ days for $\boldsymbol{P}$. maximum. The results indicate that $\boldsymbol{P}$. maximum is more sensitive to isoxaflutole than $\boldsymbol{B}$. decumbens, and that residual activity of this herbicide was longer in clay soil.
\end{abstract}

Key words: bioindicator, climatic conditions, persistence.

Recebido para publicação em 4.2.2005 e na forma revisada em 5.9.2005.

Parte da dissertação do primeiro autor apresentada para obtenção do título de Mestre em Agronomia, Área de Concentração em Proteção de Plantas.

2 Eng. Agr., M.S. Universidade Estadual de Maringá - UEM. Av. Colombo, 5790, 87020-900 Maringá-PR; ${ }^{3}$ Professor Adjunto, Departamento de Agronomia - UEM, <rsojunior@uem.br>; ${ }^{4}$ Eng.-Agr., doutoranda - UEM, Professora do Centro Integrado de Ensino Superior - CIES, Campo Mourão. ${ }^{5}$ Eng. Agr., Coordenador de Desenvolvimento de Cana-de-açúcar, Bayer CropScience. ${ }^{6}$ Graduando em Agronomia - UEM, Bolsista de Iniciação Científica. * Bolsista do CNPq.

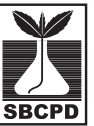

Planta Daninha, Viçosa-MG, v. 23, n. 3, p. 491-499, 2005 


\section{INTRODUÇÃO}

O isoxaflutole (IFT) é considerado um próherbicida, uma vez que rapidamente é convertido ao metabólito diquetonitrila (DKN), por meio de abertura do anel isoxazole, que é a molécula biologicamente ativa no controle de plantas daninhas (Figura 1). No entanto, sabe-se que vários fatores (clima, dose inicial e características físico-químicas do pesticida e dos solos, microrganismos) são capazes de influenciar a eficiência residual, a lixiviação e a persistência dos herbicidas. Portanto, o conhecimento dessas interações (solo x planta $\mathrm{x}$ herbicida) é determinante para que se faça uma recomendação adequada a cada tipo de solo, resultando em eficiência, economia e mínima contaminação do meio ambiente.

O IFT é sensivel à luz e à hidrólise (Lin et al., 2002); sua meia-vida em água é de 9 horas e, no solo, menor que 24 horas (Mitra et al., 2000; Lin et al., 2002). A persistência aumenta em solos secos, e sua meia-vida passa de 1,5 dia (umidade de $100 \mathrm{Kpa}$ ) para

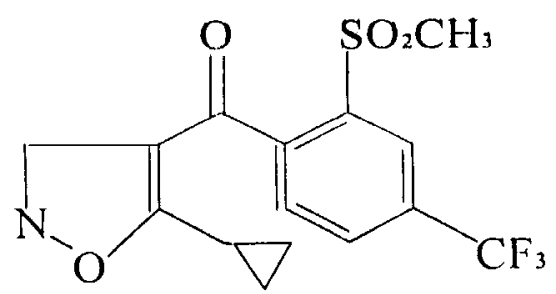

Isoxaflutole (IFT)

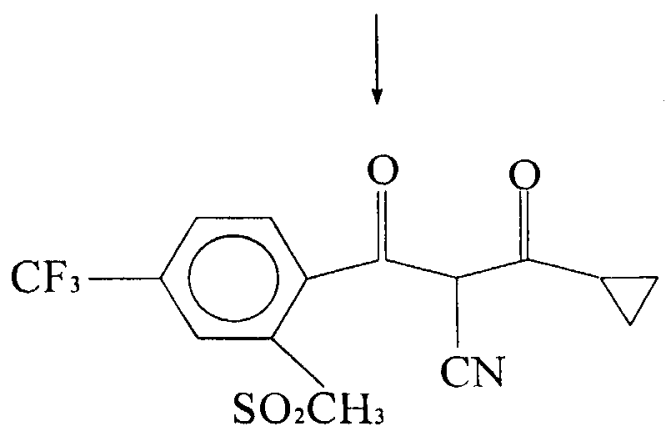

Diquetonitrila (DKN)

Fonte: Mitra et al. (1999).

Figura 1 - Estrutura química do isoxaflutole e sua forma metabolizada diquetonitrila.

Planta Daninha, Viçosa-MG, v. 23, n. 3, p. 491-499, 2005
9,6 dias (solo seco ao ar) (Taylor-Lovell et al., 2002). Segundo Vidal \& Merotto Jr. (2001), a meia-vida do IFT foi de 20 horas em solo com $30 \%$ da capacidade de campo e de 60 horas em solo com $15 \%$. A conversão de IFT em DKN é rápida no solo, na água e nas plantas (Mitra et al., 1999, 2000); no caso do solo, quanto maior a umidade, mais rápida a conversão. A meiavida diminui de 74 para 38 horas quando a umidade do solo passa de 10 para $40 \%$; se o solo estiver seco, essa reação não ocorre ou acontece muito lentamente (Taylor-Lovell et al., 2002).

A reação é química e não sofre a influência da temperatura ou dos microrganismos do solo, sendo a conversão mais rápida em solos de $\mathrm{pH}$ mais alto. Rouchaud et al. (1998) demonstraram que a dissipação é mais rápida em $\mathrm{pH} 7,2$ do que em pH 5,5. Embora tanto o IFT quanto o DKN sejam considerados moléculas nãoiônicas, é possivel haver protonação do anel isoxazole, fazendo que sua atividade seja afetada pelo $\mathrm{pH}$. O metabólito DKN é mais solúvel, mais estável e mais persistente que o IFT. Taylor-Lovell et al. (2002) relatam meiavida maior que 56 dias em solo com pH 7,0 e 2,5\% de carbono orgânico para o DKN, comparado à meia-vida menor que dois dias para o IFT no mesmo solo.

O isoxaflutole é absorvido preferencialmente pelas raízes, embora também o seja pelas sementes, o que não ocorre com seu derivado DKN, que é absorvido somente pelas raízes. Depois de absorvidos, tanto o IFT como o DKN são transportados rapidamente para o ápice da plântula, onde a maior parte do IFT é então convertida a DKN. Apesar de conversão de IFT em DKN poder ocorrer antes ou após a absorção pelas plantas (Taylor-Lovell et al., 2000), é o metabólito DKN que atua como um potente inibidor da 4-hydroxyphenylpyruvate dioxygenase (HPPD). Nas espécies tolerantes, como o milho e a cana-de-açúcar, a metabolização continua rapidamente, com a conversão do DKN em ácido benzóico (AB), considerado um metabólito, sem ação herbicida e, ao fim do processo, formando gás carbônico (Pallet et al., 1998; Sprague et al., 1999). Em espécies sensíveis, essa metabolização ocorre lentamente, permitindo a inibição enzimática do HPPD pelo DKN, bloqueando a biossintese do homogentisato, o precursor da plastoquinona e do 
$\alpha$-tocoferol (Viviani et al., 1998). O isoxaflutole bloqueia também o transporte de elétrons da fotossintese, em nivel do FSII, pela menor produção da plastoquinona necessária ao transporte de elétrons, aumentando o estresse oxidativo já provocado pela ausência da proteção dos carotenóides (Pallet et al., 1998).

Em razão de suas propriedades químicas, o isoxaflutole apresenta importante particularidade no que diz respeito à sua utilização no campo. Uma vez que a atividade herbicida depende da conversão do IFT em DKN e que esta depende da disponibilidade de água no solo, aplicações desse herbicida podem ser feitas durante todo o período mais seco do ano. Assim que se registra a ocorrência de chuvas, a conversão passa a acontecer simultaneamente à emergência das plantas daninhas, o que prolonga sua atividade residual no campo. Esse fato é particularmente interessante para as áreas de corte de cana que se concentram entre os meses de maio e agosto, em que muitos herbicidas simplesmente não são efetivos, devido à baixa disponibilidade de água no solo.

Não há informações concretas que quantifiquem o nível de umidade necessária para "ativar" o produto, nem com relação ao período que ele suporta sem umidade antes ser metabolizado. Segundo Rodrigues \& Almeida (1998), o produto apresenta boa estabilidade em condições de seca, podendo aguardar, dependendo da dose, por mais de 60 dias o início das chuvas.

Assim, é razoável admitir que a compreensão da atividade residual do IFT sob diferentes condições simuladas de seca após a aplicação pode, eventualmente, levar à aplicação de doses menores em função da(s) espécie(s) dominante(s), o que, por sua vez, implica redução do aporte de pesticidas no solo e no ambiente.

Dada a importância que o assunto vem assumindo e considerando a carência de informações, principalmente em condições tropicais, o presente trabalho teve por objetivo estudar o efeito residual de isoxaflutole sob diferentes condições de seca, após aplicação do herbicida, em dois solos de textura contrastante.

\section{MATERIAL E MÉTODOS}

Dois experimentos foram instalados em casa de vegetação no campus da Universidade Estadual de Maringá (UEM). As amostras de solos utilizadas nos experimentos foram provenientes do horizonte subsuperficial (5 a $20 \mathrm{~cm}$ ) de dois locais: Centro Técnico de Irrigação (Latossolo Vermelho distroférrico nitossólico, textura argilosa) e Fazenda Experimental de Iguatemi (Latossolo Vermelho distrófico, textura franco-argilo-arenosa) da UEM. As análises químicas e físicas das amostras dos solos encontram-se na Tabela 1 .

Após a coleta, as amostras dos solos foram peneiradas e secas ao ar. Posteriormente, esses materiais foram acondicionados em vasos plásticos com capacidade de 5 litros, sendo cada vaso uma unidade experimental. As aplicações de IFT foram feitas em préemergência, utilizando-se um pulverizador costal de pressão constante à base de $\mathrm{CO}_{2}$, com bicos tipo leque XR-110-02, pressão de $2,0 \mathrm{kgf} \mathrm{cm}^{-2}$, o que proporcionou um volume de 150 litros de calda por hectare. Por ocasião das aplicações do produto o solo encontrava-se seco.

As doses utilizadas foram de 0, 230 e $270 \mathrm{~g} \mathrm{ha}^{-1}$ nas amostras de Latossolo Vermelho distroférrico nitossólico e de 0, 180 e $200 \mathrm{~g} \mathrm{ha}^{-1}$ para o Latossolo Vermelho distrófico, conforme a recomendação de que doses mais elevadas devem ser utilizadas em solos mais argilosos e/ou ricos em matéria orgânica (Rodrigues \& Almeida, 1998). Em cada experimento, foram combinadas em esquema fatorial

Tabela 1 - Características químicas e físicas de amostras dos solos utilizadas nos experimentos

\begin{tabular}{|c|c|c|c|c|c|c|c|c|c|c|c|c|}
\hline \multirow{2}{*}{ Solo } & \multicolumn{2}{|c|}{$\mathrm{pH}$} & $\mathrm{Al}^{3+}$ & $\mathrm{H}^{+}+\mathrm{Al}^{3+}$ & $\mathrm{Ca}^{+2}+\mathrm{Mg}^{2+}$ & $\mathrm{Ca}^{2+}$ & $\mathrm{K}^{+}$ & $P$ & C & Areia & Silte & Argila \\
\hline & $\left(\mathrm{CaCl}_{2}\right)$ & $\left(\mathrm{H}_{2} \mathrm{O}\right)$ & \multicolumn{5}{|c|}{$\left(\mathrm{cmol}_{\mathrm{c}} \mathrm{dm}^{-3}\right)$} & \multicolumn{2}{|c|}{$\left(\mathrm{mg} \mathrm{dm}^{-3}\right)$} & \multicolumn{3}{|c|}{$(\%)$} \\
\hline $\mathrm{LV}^{1 /}$ & 4,1 & 6,0 & 0,2 & 3,68 & 9,97 & 6,75 & 0,24 & 1 & 10,36 & 12 & 16 & 72 \\
\hline $\mathrm{LR}^{2 /}$ & 4,0 & 4,9 & 0,7 & 3,42 & 1,64 & 1,03 & 0,07 & 1 & 3,07 & 65 & 8 & 27 \\
\hline
\end{tabular}

${ }^{1 /} \mathrm{LV}=$ Latossolo Vermelho distroférrico nitossólico (textura argilosa). ${ }^{2 /} \mathrm{LR}=$ Latossolo Vermelho distrófico (textura franco-argilo-arenosa). 
três doses do herbicida, duas espécies bioindicadoras (Brachiaria decumbens e Panicum maximum) e sete períodos de seca após as aplicações do IFT (0, 20, 40, 60, 80, 100 e 120 dias após a aplicação - DAA). Ambos os experimentos foram conduzidos em delineamentos inteiramente casualizados, com quatro repetições. Os períodos de seca referem-se ao período de tempo, a partir da aplicação do produto, em que os vasos foram mantidos sem irrigação ou semeadura do bioindicador. Transcorridos os períodos de tempo previamente estabelecidos, foi realizada a semeadura dos bioindicadores (1,5 $\mathrm{cm}$ de profundidade e $10 \mathrm{~g}$ de sementes por vaso) e, imediatamente após, aplicada uma irrigação equivalente a uma lâmina de $20 \mathrm{~mm}$. Essa intensidade de precipitação é considerada suficiente para ativar os produtos que são aplicados no solo em áreas de plantio direto (Velini et al., 2002).

Depois de aplicada a lâmina de água, os vasos foram mantidos com irrigação diária até o encerramento das avaliações, aos 60 dias após a semeadura (DAS) dos bioindicadores. As avaliações de controle foram realizadas aos 15 , 30,45 e 60 DAS, por meio de atribuição de porcentagem de controle (escala visual 0-100\%), em relação às plantas que não receberam aplicação de herbicidas (SBCPD, 1995).

Os dados de controle do bioindicador foram analisados por meio de análise de variância e de regressão, utilizando-se o pacote estatístico SAEG (1997).

\section{RESULTADOS E DISCUSSÃO}

\section{Latossolo Vermelho distroférrico nitossólico (textura argilosa)}

Embora tenha havido interação significativa entre os fatores dose e periodo, as médias de controle de ambos os bioindicadores foram, para todas as épocas de avaliação, superiores a 97\% no Latossolo Vermelho distroférrico nitossólico (Tabela 2). Com isso, tornou-se irrelevante ajustar curvas de regressão tanto para $B$. decumbens quanto para $P$. maximum. O resultado obtido no solo Latossolo Vermelho distroférrico nitossólico assemelhase a dados de trabalhos instalados a campo, em que se observa acima de $80 \%$ de controle por períodos de até 80 dias para espécies de plantas daninhas, como B. decumbens, B. plantaginea, Digitaria horizontalis, Portulaca oleracea, Eleusine indica, Cenchrus echinatus, Galinsoga parviflora, Amaranthus viridis e Ageratum conyzoides (Constantin et al., 2000; Costa et al., 2000, 2002a, b; Adoryan et al., 2002a, b; Montório et al., 2002). Kruse et al. (2001) também constataram, igualmente, controles em torno de 90\% para Abutilon theophrasti, Ipomoea hederacea e $A$. retroflexus aos 52 dias após a aplicação de isoxaflutole na dose de 52,5 $\mathrm{g} \mathrm{ha}^{-1}$ em um solo franco-argilo-siltoso, com 3,5\% de matéria orgânica e $\mathrm{pH}$ 6,3. Com base nos

Tabela 2 - Porcentagens de controle (médias, \pm desvio-padrão) aos 15, 30, 45 e 60 dias após a semeadura do bioindicador, em amostras de Latossolo Vermelho distroférrico nitossólico

\begin{tabular}{|c|c|c|c|c|}
\hline \multirow{2}{*}{$\begin{array}{c}\text { Dias após a } \\
\text { aplicação }\end{array}$} & \multicolumn{4}{|c|}{ Épocas de avaliação (dias após a semeadura) } \\
\hline & 15 DAS & 30 DAS & 45 DAS & 60 DAS \\
\hline \multicolumn{5}{|c|}{ Brachiaria decumbens } \\
\hline \multicolumn{5}{|c|}{ Dose $=230 \mathrm{~g} \mathrm{ha}^{-1}$} \\
\hline 0 & $99,75 \pm 0,96$ & $100,00 \pm 0,00$ & $97,75 \pm 2,50$ & $98,75 \pm 1,26$ \\
\hline 20 & $99,75 \pm 0,50$ & $99,75 \pm 0,50$ & $100,00 \pm 0,00$ & $100,00 \pm 0,00$ \\
\hline 40 & $99,25 \pm 0,96$ & $99,00 \pm 0,82$ & $99,00 \pm 1,41$ & $99,50 \pm 1,00$ \\
\hline 60 & $99,75 \pm 0,50$ & $100,00 \pm 0,00$ & $100,00 \pm 0,00$ & $100,00 \pm 0,00$ \\
\hline 80 & $99,75 \pm 0,50$ & $100,00 \pm 0,00$ & $100,00 \pm 0,00$ & $100,00 \pm 0,00$ \\
\hline 100 & $100,00 \pm 0,00$ & $100,00 \pm 0,00$ & $99,50 \pm 0,58$ & $100,00 \pm 0,00$ \\
\hline 120 & $100,00 \pm 0,00$ & $100,00 \pm 0,00$ & $100,00 \pm 0,00$ & $99,75 \pm 0,50$ \\
\hline \multicolumn{5}{|c|}{ Dose $=270 \mathrm{~g} \mathrm{ha}^{-1}$} \\
\hline 0 & $100,00 \pm 0,00$ & $100,00 \pm 0,00$ & $100,00 \pm 0,00$ & $100,00 \pm 0,00$ \\
\hline 20 & $99,75 \pm 0,50$ & $99,25 \pm 0,96$ & $100,00 \pm 0,00$ & $100,00 \pm 0,00$ \\
\hline 40 & $99,75 \pm 0,50$ & $99,75 \pm 0,50$ & $100,00 \pm 0,00$ & $100,00 \pm 0,00$ \\
\hline 60 & $99,50 \pm 0,58$ & $100,00 \pm 0,00$ & $100,00 \pm 0,00$ & $100,00 \pm 0,00$ \\
\hline 80 & $99,50 \pm 0,58$ & $100,00 \pm 0,00$ & $100,00 \pm 0,00$ & $100,00 \pm 0,00$ \\
\hline 100 & $100,00 \pm 0,00$ & $100,00 \pm 0,00$ & $99,75 \pm 0,50$ & $100,00 \pm 0,00$ \\
\hline 120 & $100,00 \pm 0,00$ & $100,00 \pm 0,00$ & $100,00 \pm 0,00$ & $99,75 \pm 0,50$ \\
\hline \multicolumn{5}{|c|}{ Panicum maximum } \\
\hline \multicolumn{5}{|c|}{ Dose $=230 \mathrm{~g} \mathrm{ha}^{-1}$} \\
\hline 0 & $99,25 \pm 0,50$ & $100,00 \pm 0,00$ & $100,00 \pm 0,00$ & $99,00 \pm 1,41$ \\
\hline 20 & $100,00 \pm 0,00$ & $100,00 \pm 0,00$ & $99,25 \pm 0,50$ & $99,75 \pm 0,50$ \\
\hline 40 & $99,25 \pm 0,96$ & $99,75 \pm 0,50$ & $99,50 \pm 0,58$ & $99,75 \pm 0,50$ \\
\hline 60 & $99,25 \pm 0,50$ & $100,00 \pm 0,00$ & $100,00 \pm 0,00$ & $100,00 \pm 0,00$ \\
\hline 80 & $100,00 \pm 0,00$ & $100,00 \pm 0,00$ & $100,00 \pm 0,00$ & $100,00 \pm 0,00$ \\
\hline 100 & $100,00 \pm 0,00$ & $100,00 \pm 0,00$ & $99,75 \pm 0,50$ & $100,00 \pm 0,00$ \\
\hline 120 & $100,00 \pm 0,00$ & $100,00 \pm 0,00$ & $100,00 \pm 0,00$ & $100,00 \pm 0,00$ \\
\hline \multicolumn{5}{|c|}{ Dose $=270 \mathrm{~g} \mathrm{ha}^{-1}$} \\
\hline 0 & $100,00 \pm 0,00$ & $99,25 \pm 0,96$ & $100,00 \pm 0,00$ & $99,75 \pm 0,50$ \\
\hline 20 & $98,50 \pm 2,38$ & $100,00 \pm 0,00$ & $100,00 \pm 0,00$ & $100,00 \pm 0,00$ \\
\hline 40 & $99,75 \pm 0,50$ & $100,00 \pm 0,00$ & $99,75 \pm 0,50$ & $100,00 \pm 0,00$ \\
\hline 60 & $99,50 \pm 0,58$ & $100,00 \pm 0,00$ & $100,00 \pm 0,00$ & $100,00 \pm 0,00$ \\
\hline 80 & $\begin{array}{l}100,00 \pm 0,00 \\
0.000\end{array}$ & $\begin{array}{l}100,00 \pm 0,00 \\
\cdots \\
0.03\end{array}$ & $100,00 \pm 0,00$ & $100,00 \pm 0,00$ \\
\hline 100 & $100,00 \pm 0,00$ & $100,00 \pm 0,00$ & $100,00 \pm 0,00$ & $100,00 \pm 0,00$ \\
\hline 120 & $100,00 \pm 0,00$ & $100,00 \pm 0,00$ & $100,00 \pm 0,00$ & $100,00 \pm 0,00$ \\
\hline
\end{tabular}

Os valores após \pm referem-se ao desvio-padrão das médias. 
resultados obtidos neste trabalho e nos dados experimentais da literatura, fica evidente que o efeito residual de IFT pode ser considerado longo em solos de textura mais argilosa e com maior teor de carbono orgânico (CO). Esses resultados devem-se provavelmente ao fato de que as argilas, juntamente com a matéria orgânica, apresentam grande superfície específica e elevada capacidade de retenção e trocas iônicas - propriedades que proporcionam menor exposição do produto aos processos de degradação e transporte, o que prolongou o efeito residual do IFT no solo de textura argilosa.

\section{Latossolo Vermelho distrófico (textura franco-argilo-arenosa)}

Pelo teste $\mathrm{F}$ em nivel de $5 \%$, verificou-se que foram significativos os efeitos tanto dos fatores isolados como da interação entre eles para o Latossolo Vermelho distrófico. Os efeitos de períodos /doses (exceto para dose zero) foram descritos por meio de regressões. As médias de controle de $B$. decumbens e $P$. maximum encontram-se na Tabela 3.

Ajustadas as equações de regressão (Tabela 4), foi constatado em todas as avaliações de controle que, na dose de $180 \mathrm{~g} \mathrm{ha}^{-1}$ (Figura 2), houve redução no controle à medida que se aumentou o período de seca após a aplicação do produto no solo. Essa redução pode estar ligada à degradação do produto no solo, fazendo com que seu efeito residual seja reduzido à medida que se prolonga a exposição do produto aos processos de transporte e degradação. Comparando as duas espécies bioindicadoras, observa-se maior suscetibilidade ao isoxaflutole de $P$. maximum, considerando a avaliação aos 15 DAS (Figura 2A). Para esta espécie, obteve-se controle maior ou igual a $80 \%$ com períodos de seca após a aplicação de até 50 dias (dados calculados pela equação de regressão); para $B$. decumbens o controle $\geq 80 \%$ ocorreu apenas para períodos de seca de até 29 dias após a aplicação. A velocidade de metabolização do IFT é considerada a base da seletividade do produto. Espécies tolerantes, como o milho, possuem a capacidade genética de degradar rapidamente o IFT e o DKN em AB (Franssen et al., 2001). Pallet et al. (1998) demonstraram que a conversão de DKN em AB chega a $59 \%$ num período de seis dias após a aplicação de IFT em plantas de milho (tolerante), comparada a apenas $12 \%$ em A. theophrasti (suscetivel), ao passo que, no mesmo periodo, $29 \%$ do total de ${ }^{14} \mathrm{C}$-IFT permaneceu na forma de DKN em milho e $82 \%$ em $A$. theophrasti. É provável que $B$. decumbens e $P$. maximum apresentem capacidades diferenciadas de converter o DKN em $\mathrm{AB}$, o que explicaria a maior suscetibilidade deste último em relação a $B$. decumbens.

Aos 30 DAS (Figura 2B), houve tendência de melhor controle para $B$. decumbens, que se estendeu até 27 dias de seca (dados calculados pela equação de regressão) após aplicação, na

Tabela 3 - Porcentagens de controle (médias, \pm desvio-padrão) aos 15, 30, 45 e 60 dias após a semeadura do bioindicador, em amostras de Latossolo Vermelho distrófico

\begin{tabular}{|c|c|c|c|c|}
\hline \multirow{2}{*}{$\begin{array}{c}\text { Dias após a } \\
\text { aplicação }\end{array}$} & \multicolumn{4}{|c|}{ Épocas de avaliação (dias após a semeadura) } \\
\hline & 15 DAS & $30 \mathrm{DAS}$ & 45 DAS & 60 DAS \\
\hline \multicolumn{5}{|c|}{ Brachiaria decumbens } \\
\hline \multicolumn{5}{|c|}{ Dose $=180 \mathrm{~g} \mathrm{ha}^{-1}$} \\
\hline 0 & $100,00 \pm 0,00$ & $100,00 \pm 0,00$ & $100,00 \pm 0,00$ & $100,00 \pm 0,00$ \\
\hline 20 & $96,75 \pm 1,26$ & $99,00 \pm 1,41$ & $98,50 \pm 2,38$ & $98,50 \pm 2,38$ \\
\hline 40 & $62,50 \pm 10,41$ & $84,00 \pm 13,83$ & $84,25 \pm 13,33$ & $92,00 \pm 8,52$ \\
\hline 60 & $66,75 \pm 2,36$ & $52,50 \pm 2,89$ & $33,75 \pm 2,50$ & $66,00 \pm 6,16$ \\
\hline 80 & $36,25 \pm 2,50$ & $21,25 \pm 6,29$ & $25,00 \pm 4,08$ & $11,25 \pm 4,79$ \\
\hline 100 & $48,75 \pm 18,87$ & $52,50 \pm 20,62$ & $41,25 \pm 39,45$ & $32,50 \pm 20,62$ \\
\hline 120 & $32,50 \pm 2,89$ & $51,25 \pm 4,79$ & $38,75 \pm 10,31$ & $22,50 \pm 9,57$ \\
\hline \multicolumn{5}{|c|}{ Dose $=270 \mathrm{~g} \mathrm{ha}^{-1}$} \\
\hline 0 & $99,50 \pm 0,58$ & $100,00 \pm 0,00$ & $100,00 \pm 0,00$ & $100,00 \pm 0,00$ \\
\hline 20 & $96,50 \pm 0,58$ & $99,00 \pm 1,15$ & $99,50 \pm 0,58$ & $99,50 \pm 0,58$ \\
\hline 40 & $65,50 \pm 7,14$ & $82,25 \pm 8,22$ & $75,75 \pm 10,90$ & $80,50 \pm 14,64$ \\
\hline 60 & $61,75 \pm 3,95$ & $25,00 \pm 4,08$ & $65,00 \pm 15,81$ & $56,25 \pm 9,46$ \\
\hline 80 & $72,50 \pm 11,90$ & $56,25 \pm 25,62$ & $73,25 \pm 17,29$ & $55,00 \pm 19,15$ \\
\hline 100 & $56,25 \pm 9,46$ & $58,70 \pm 11,81$ & $67,00 \pm 18,42$ & $67,50 \pm 18,48$ \\
\hline 120 & $37,50 \pm 11,90$ & $31,25 \pm 27,20$ & $26,25 \pm 19,74$ & $62,25 \pm 28,29$ \\
\hline \multicolumn{5}{|c|}{ Panicum maximum } \\
\hline \multicolumn{5}{|c|}{ Dose $=180 \mathrm{~g} \mathrm{ha}^{-1}$} \\
\hline 0 & $99,00 \pm 2,00$ & $100,00 \pm 0,00$ & $\begin{array}{l}100,00 \pm 0,00 \\
0\end{array}$ & $100,00 \pm 0,00$ \\
\hline 20 & $90,50 \pm 7,59$ & $99,25 \pm 0,96$ & $99,50 \pm 0,58$ & $99,75 \pm 0,50$ \\
\hline 40 & $79,50 \pm 4,20$ & $93,25 \pm 4,99$ & $94,75 \pm 3,30$ & $98,50 \pm 1,29$ \\
\hline 60 & $74,25 \pm 7,23$ & $94,00 \pm 2,00$ & $94,50 \pm 1,73$ & $90,00 \pm 0,00$ \\
\hline 80 & $61,25 \pm 7,50$ & $84,50 \pm 6,40$ & $80,00 \pm 21,21$ & $86,25 \pm 14,36$ \\
\hline 100 & $86,50 \pm 24,34$ & $87,00 \pm 24,67$ & $81,25 \pm 34,17$ & $90,00 \pm 13,54$ \\
\hline 120 & $45,00 \pm 30,00$ & $85,75 \pm 12,47$ & $86,00 \pm 8,98$ & $87,50 \pm 10,31$ \\
\hline \multicolumn{5}{|c|}{ Dose $=200 \mathrm{~g} \mathrm{ha}^{-1}$} \\
\hline 0 & $100,00 \pm 0,00$ & $100,00 \pm 0,00$ & $100,00 \pm 0,00$ & $100,00 \pm 0,00$ \\
\hline 20 & $97,50 \pm 2,89$ & $100,00 \pm 0,00$ & $100,00 \pm 0,00$ & $100,00 \pm 0,00$ \\
\hline 40 & $91,75 \pm 5,38$ & $94,50 \pm 4,12$ & $89,50 \pm 10,97$ & $92,25 \pm 8,38$ \\
\hline 60 & $94,25 \pm 3,86$ & $98,25 \pm 0,96$ & $97,25 \pm 1,71$ & $97,75 \pm 0,96$ \\
\hline 80 & $60,00 \pm 20,82$ & $92,25 \pm 8,26$ & $86,25 \pm 17,75$ & $94,50 \pm 3,70$ \\
\hline 100 & $94,75 \pm 3,30$ & $97,00 \pm 0,82$ & $59,50 \pm 30,78$ & $93,25 \pm 11,64$ \\
\hline 120 & $83,00 \pm 12,62$ & $77,50 \pm 28,72$ & $86,00 \pm 18,64$ & $77,25 \pm 28,46$ \\
\hline
\end{tabular}

Os valores após \pm referem-se ao desvio-padrão das médias.

Planta Daninha, Viçosa-MG, v. 23, n. 3, p. 491-499, 2005 
Tabela 4 - Equações da regressão obtidas para cada dose dentro de todos os períodos, em amostras de Latossolo Vermelho distrófico

\begin{tabular}{|c|c|c|c|c|c|}
\hline Espécie & Dose & DAS & $\mathrm{R}^{2}$ & $Y=a+b x+c x^{2}$ & $\begin{array}{l}\text { Duração do efeito de } \\
\text { controle para } \mathrm{Y}=80\end{array}$ \\
\hline B. decumbens & 180 & 15 & 0,88 & $104,60-(0,9343 \mathrm{P})+\left(0,00295 \mathrm{P}^{2}\right)$ & 29 \\
\hline P. maximum & 180 & 15 & 0,62 & $96,78-(0,3388 \mathrm{P})+\left(0,00002 \mathrm{P}^{2}\right)$ & 50 \\
\hline B. decumbens & 200 & 15 & 0,82 & $99,16-(0,5491 \mathrm{P})+\left(0,00071 \mathrm{P}^{2}\right)$ & 37 \\
\hline P. maximum & 200 & 15 & 0,31 & $103,13-(0,4531 \mathrm{P})+\left(0,00246 \mathrm{P}^{2}\right)$ & $>120$ \\
\hline B. decumbens & 180 & 30 & 0,75 & $111,86-(1,3625 \mathrm{P})+\left(0,00686 \mathrm{P}^{2}\right)$ & 27 \\
\hline P. maximum & 180 & 30 & 0,87 & $101,26-(0,2054 \mathrm{P})+\left(0,00058 \mathrm{P}^{2}\right)$ & $>120$ \\
\hline B. decumbens & 200 & 30 & 0,67 & $106,53-(1,0612 \mathrm{P})+\left(0,00419 \mathrm{P}^{2}\right)$ & 28 \\
\hline P. maximum & 200 & 30 & 0,68 & $98,42+(0,0996 \mathrm{P})-\left(0,00196 \mathrm{P}^{2}\right)$ & 73 \\
\hline B. decumbens & 180 & 45 & 0,81 & $112,23-(1,4634 \mathrm{P})+\left(0,00686 \mathrm{P}^{2}\right)$ & 25 \\
\hline P. maximum & 180 & 45 & 0,76 & $102,50-(0,2656 \mathrm{P})+\left(0,00083 \mathrm{P}^{2}\right)$ & $>120$ \\
\hline B. decumbens & 200 & 45 & 0,82 & $98,82-(0,2450 \mathrm{P})-\left(0,00225 \mathrm{P}^{2}\right)$ & 50 \\
\hline P. maximum & 200 & 45 & 0,60 & $100,36-(0,1576 \mathrm{P})+\left(0,00041 \mathrm{P}^{2}\right)$ & $>120$ \\
\hline B. decumbens & 180 & 60 & 0,82 & $110,04-(0,9335 \mathrm{P})+\left(0,00115 \mathrm{P}^{2}\right)$ & 33 \\
\hline P. maximum & 180 & 60 & 0,83 & $98,73-(0,1308 \mathrm{P})-\left(0,00332 \mathrm{P}^{2}\right)$ & 58 \\
\hline B. decumbens & 200 & 60 & 0,83 & $106,85-(1,0040 \mathrm{P})+\left(0,00535 \mathrm{P}^{2}\right)$ & 32 \\
\hline P. maximum & 200 & 60 & 0,87 & $98,93+(0,0544 \mathrm{P})-\left(0,00193 \mathrm{P}^{2}\right)$ & 114 \\
\hline
\end{tabular}

Em todas as equações, $\mathrm{Y}=$ controle visual (\%) e $\mathrm{P}=$ período de seca após a aplicação (dias).

dose de $180 \mathrm{~g}^{\text {ha-1 }}$ de isoxaflutole. É possível que essa melhora de controle se deva à metabolização do IFT a DKN durante esse intervalo, fazendo com que o controle fosse efetivo até o período de 27 dias. Para $P$. maximum, os dados indicam realmente a maior suscetibilidade da planta ao produto, pois houve controle efetivo (acima de 80\%) na dose de $180 \mathrm{~g} \mathrm{ha}^{-1}$ de IFT em um período maior que 120 dias. Relatos de Taylor-Lovell et al. (2002) mostram que três dias após o tratamento com isoxaflutole apenas $1 \%$ do herbicida aplicado havia sido convertido em $\mathrm{AB}$ e que, 56 dias após o tratamento, essa porcentagem foi elevada somente para $17 \%$, sugerindo que a lenta passagem de DKN para AB poderia contribuir para o evidente efeito residual do produto, o que pode indicar, para o solo em questão, que as porcentagens de DKN aos 27 dias de seca após a última chuva ainda permaneciam suficientemente elevadas, em niveis que controlavam $B$. decumbens. Essa porcentagem de DKN no solo foi suficiente para controlar P. maximum por mais de 120 dias de seca após a precipitação de $20 \mathrm{~mm}$. Padrões semelhantes de controle de $B$. decumbens e $P$. maximum para a dose de $180 \mathrm{~g} \mathrm{ha}^{-1}$ repetemse nas avaliações realizadas aos 45 e 60 DAS dos bioindicadores no solo Latossolo Vermelho distrófico (Figura 2C e D).

Aos 15 DAS (200 $\mathrm{g} \mathrm{ha}^{-1}$ ) de IFT (Figura 3A), para $B$. decumbens, a tendência foi a mesma da dose de $180 \mathrm{~g} \mathrm{ha}^{-1}$, mantendo-se acima de $80 \%$ de controle até os 37 dias de seca após a aplicação. Para $P$. maximum, esse efeito de controle estendeu-se além dos 120 dias de seca após a aplicação.

$\mathrm{Na}$ avaliação de 30 DAS (200 g ha ${ }^{-1}$ ) de IFT (Figura 3B), B. decumbens permaneceu praticamente com o mesmo efeito da dose de $180 \mathrm{~g} \mathrm{ha}^{-1}$, sendo controlada $(\geq 80 \%)$ até o período de 28 dias, ao passo que para $P$. maximum o controle foi obtido por 73 dias.

Ao se observar a curva de controle aos 45 DAS (Figura 3C) na dose de $200 \mathrm{~g} \mathrm{ha}^{-1}$, verificou-se praticamente a mesma tendência mostrada aos 30 DAS para ambas as espécies estudadas, sendo o controle de $B$. decumbens efetivo até o período de 50 dias de seca, e o de $P$. maximum, por um período maior que 120 dias.

Foi constatado para $B$. decumbens, aos 60 DAS (Figura 3D), que o controle efetivo (acima de $80 \%$ ) foi obtido somente até o período de 
32 dias de seca. Entretanto, para P. maximum, obteve-se controle acima de $80 \%$ até o período de 114 dias de seca, podendo-se concluir que foi obtido controle considerável após as condições impostas ao produto. As diferenças observadas no controle inicial (15 DAS) para o controle final (60 DAS) podem ser explicadas pela diferença na germinação das sementes de plantas daninhas utilizadas e também pelo tempo em que o produto permaneceu em contato com a umidade, pois, segundo Beltran et al.
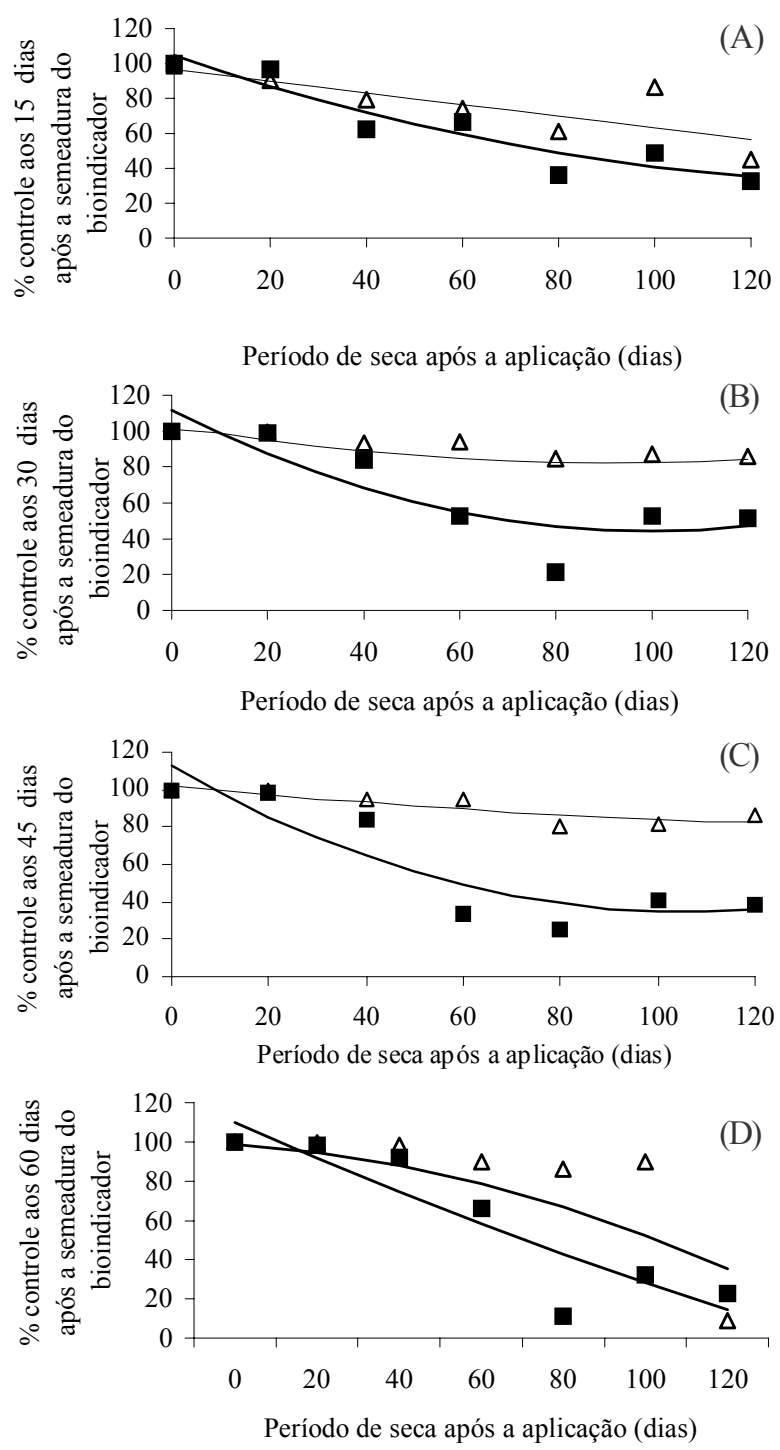

Figura 2 - Controle de $B$. decumbens ( $\square)$ e P. maximum $(\Delta)$ aos 15 (A), 30 (B), 45 (C) e 60 (D) dias após a semeadura (DAS), para a dose de $180 \mathrm{~g} \mathrm{ha}^{-1}$ de isoxaflutole aplicado em amostras de Latossolo Vermelho distrófico.
(2003) e Taylor-Lovell et al. (2002), a hidrólise de IFT para DKN é rápida e depende de $\mathrm{pH}$ e da umidade do solo e o processo químico da conversão de DKN em AB aparentemente é essencialmente ligado à atividade biológica do solo. É possivel que nesse período de 60 DAS, já tendo passado pelo período de seca imposto, tenha havido diminuição do efeito de controle de $P$. maximum na menor dose, fazendo com que se obtivesse controle maior que $80 \%$, até o período de 58 dias de seca.
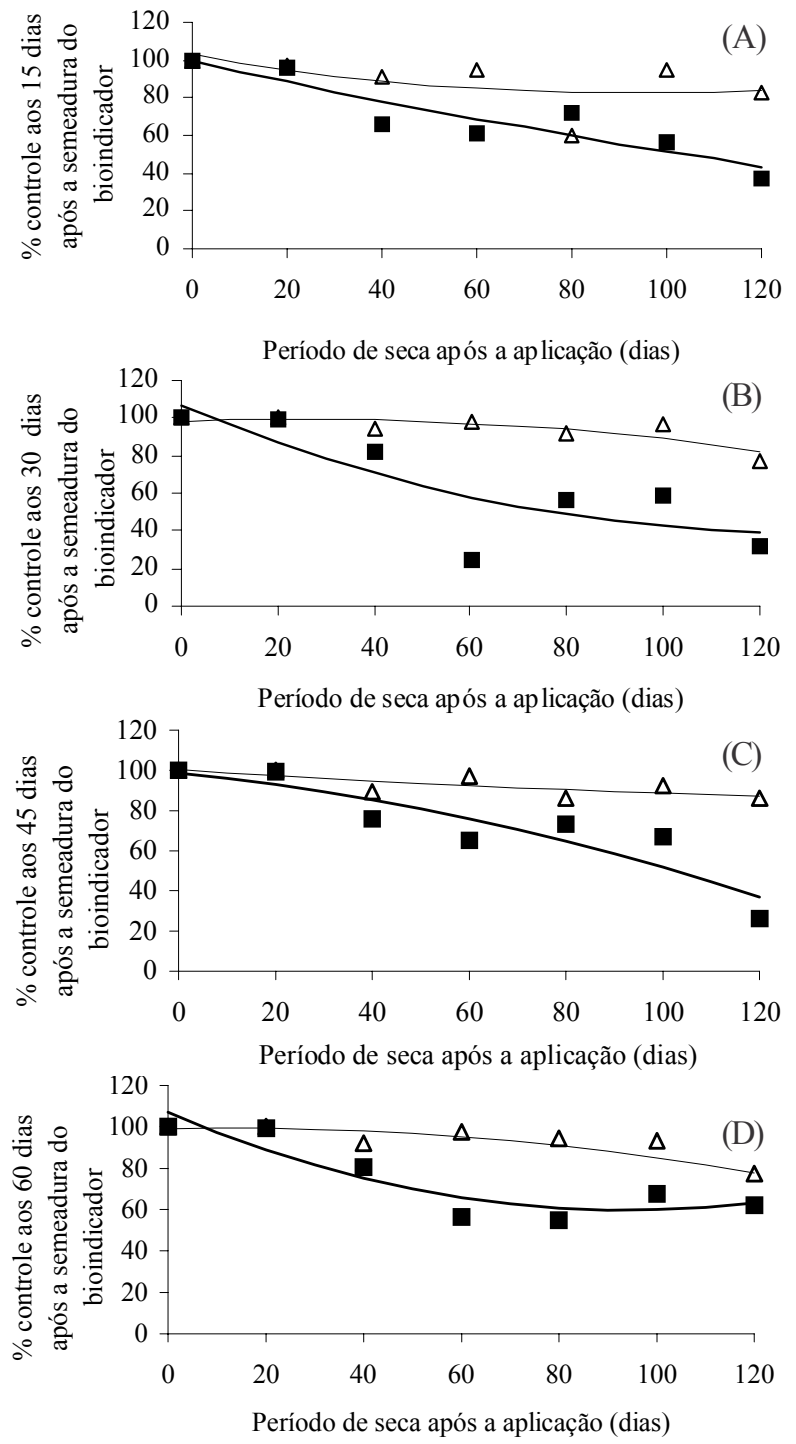

Figura 3 - Controle de $B$. decumbens ( $\mathbf{\square})$ e P. maximum $(\Delta)$ aos 15 (A), 30 (B), 45 (C) e 60 (D) dias após a semeadura (DAS), para a dose de $200 \mathrm{~g} \mathrm{ha}^{-1}$ de isoxaflutole aplicado em amostras de Latossolo Vermelho distrófico.

Planta Daninha, Viçosa-MG, v. 23, n. 3, p. 491-499, 2005 
Ao comparar os dois solos, verificou-se que, independentemente da dose, do bioindicador e do período de seca avaliados, o IFT apresentou maior efeito residual no solo de textura argilosa. Esse fato, aliado às grandes diferenças nos teores de $\mathrm{CO}$ e argila entre os dois solos (Tabela 1), indica claramente que os mecanismos de retenção foram mais fortes e, conseqüentemente, mais estáveis nas amostras de Latossolo Vermelho distroférrico nitossólico. Por sua vez, o Latossolo Vermelho distrófico possui características (menor quantidade de sitios sortivos disponiveis, por exemplo) que conferem a menor sorção de IFT nas amostras estudadas.

Contudo, para elucidar esses aspectos, há necessidade de desenvolver outros trabalhos referentes à determinação da persistência e mobilidade, a fim de complementar os estudos de efeito residual no solo desse herbicida.

\section{LITERATURA CITADA}

ADORYAN, M. L.; BENDECK, O. B.; GELMINI, G. A. Avaliação do herbicida isoxaflutole na cultura de Pinus caribae. In: CONGRESSO BRASILEIRO DA CIÊNCIA DAS PLANTAS DANINHAS, 23., 2002, Gramado. Resumos... Londrina: SBCPD/Embrapa Clima Temperado, 2002a. p. 577.

ADORYAN, M. L.; BENDECK, O. B.; GELMINI, G. A. Eficácia e seletividade do herbicida isoxaflutole na cultura de Eucalyptus grandis. In: CONGRESSO BRASILEIRO DA CIÊNCIA DAS PLANTAS DANINHAS, 23., 2002,

Gramado. Resumos... Londrina: SBCPD/Embrapa Clima Temperado, 2002b. p. 576.

BELTRAN, E. et al. Fate of isoxaflutole in soil under controlled conditions. J. Agric. Food Chem., v. 51, p. 146-151, 2003.

CONSTANTIN, J.; OLIVEIRA JR., R. S.; MACIEL, C. D. G. Isoxaflutole: controle de Brachiaria decumbens e seletividade para cana-soca. In: CONGRESSO BRASILEIRO DA CIÊNCIA DAS PLANTAS DANINHAS, 22., 2000, Foz do Iguaçu. Resumos... Londrina: SBCPD, 2000. p. 297.

COSTA, E. A. D.; GELMINI, G. A.; ZAMBON, S

Avaliação de isoxaflutole aplicado isoladamente ou em mistura de tanque no nivelamento do solo para controle pré-emergente de infestantes em cana-planta. In: CONGRESSO BRASILEIRO DA CIÊNCIA DAS PLANTAS DANINHAS, 22., 2000, Foz do Iguaçu. Resumos... Londrina: SBCPD, 2000. p. 295.

Planta Daninha, Viçosa-MG, v. 23, n. 3, p. 491-499, 2005
COSTA, E. A. D.; MATALlO, M. B.; MACEDO, E. C. Eficiência de isoxaflutole aplicado em pré-emergência de plantas daninhas em área reflorestada com pinus. In: CONGRESSO BRASILEIRO DA CIÊNCIA DAS PLANTAS DANINHAS, 23., 2002, Gramado. Resumos... Londrina: SBCPD/Embrapa Clima Temperado, 2002a. p. 560 .

COSTA, E. A. D. et al. Eficiência de isoxaflutole em solo Latossolo Vermelho distroférrico nitossólico aplicado em préemergência de plantas daninhas em área reflorestada com eucalipto. In: CONGRESSO BRASILEIRO DA CIÊNCIA DAS PLANTAS DANINHAS, 23., 2002, Gramado. Resumos... Londrina: SBCPD/Embrapa Clima Temperado, 2002b. p. 561.

FRANSSEN, A. S.; DOUCHES, D. S.; KELLS, J. J. Inheritance of isoxaflutole tolerance in corn. North Central Weed Sci. Soc. Abstr. 56. [CD-Rom Computer file]. North Central Weed Sci. Soc., Champaign: 2001.

KRUSE, N. D. et al. Sinergismo potencial entre herbicidas inibidores do fotossistema II e da síntese de carotenóides. Ci. Rural, v. 31, n. 4, p. 569-575, 2001.

LIN, C. H. et al. Determination of isoxaflutole (Balance) and its metabolites in water using solid phase extraction followed by high-performance liquid chromatography with ultraviolet or mass spectrometry. J.Agric. Food Chem., v. 50, p. 5816-5824, 2002.

MITRA, S.; BHOWMILK, P. C.; XING, B. Sorption and desorption of the diketonitrile metabolite of isoxaflutole in soils. Environ. Poll., v. 108, p. 183-190, 2000.

MITRA, S.; BHOWMILK, P. C.; XING, B. Sorption of isoxaflutole by five different soils varying in physical and chemical properties. Pestic. Sci., v. 55, p. 935-942, 1999.

MONTÓRIO, G. A. et al. Avaliação da eficiência agronômica de herbicidas aplicados em área de alta infestação de capimcolchão (Digitaria horizontalis Willd) na cultura de cana-deaçúcar. In: CONGRESSO BRASILEIRO DA CIÊNCIA DAS PLANTAS DANINHAS, 23., 2002, Gramado. Resumos... Londrina: SBCPD/Embrapa Clima Temperado, 2002. p. 508.

PALLET, K. E. et al. The mode of action of isoxaflutole. I. Physiological effects, metabolism, and selectivity. Pestic. Bioch. Physiol., v. 62, p. 113-124, 1998.

RODRIGUES, B. N.; ALMEIDA, F. S. Guia de herbicidas. 4.ed. Londrina: Edição dos Autores, 1998. 648 p.

ROUCHAUD, J. et al. Isoxaflutole herbicide persistence and mobility in summer corn and winter wheat crops. Bull.

Environ. Contam. Toxicol., v. 60, p. 577-584, 1998.

SAEG - Sistema para Análises Estatísticas, versão 7.0, Viçosa: Fundação Arthur Bernardes, 1997. 
SOCIEDADE BRASILEIRA DA CIÊNCIA DAS PLANTAS DANINHAS - SBCPD. Procedimentos para instalação, avaliação e análise de experimentos com herbicidas. Londrina: $1995.42 \mathrm{p}$.

SPRAGUE, C. L.; PENNER, D.; KELLS, J. J. Physiological basis for tolerance of four Zea mays hybrids to RPA 201772. Weed Sci., v. 47, p. 613-635, 1999.

TAYLOR-LOVELL, S. et al. Hydrolisis and soil adsorption of the labile herbicide isoxaflutole. Envion. Sci. Technol., v. 34, p. 3186-3190, 2000.

TAYLOR-LOVELL, S.; SIMS, G. K.; WAX, L. M. Effects of moisture, temperature, and biological activity on the degradation of isoxaflutole in soil. J. Agric. Food Chem., v. 50, p. 5626-5633, 2002.
VELINI, E. D. et al. Dinâmica de tebuthiuron em palhadas de cana-de-açúcar. Efeito de lâminas de chuva, surfactantes e volumes simulados de aplicação. In: CONGRESSO BRASILEIRO DA CIÊNCIA DAS PLANTAS DANINHAS, 23., 2002, Gramado. Resumos. Londrina: SBCPD/Embrapa Clima Temperado, 2002. p. 705.

VIDAL, R. A.; MEROTTO JR., A. Herbicidologia. Porto Alegre: Edição dos Autores, 2001. 75 p.

VIVIANI, F.; LITTLE, J. P.; PALLET, K. E. The mode of action of isoxaflutole. II. Characterization of the inhibition of carrot 4-hydroxyphenylpyruvate dioxygenase by the diketonitrile derivative of isoxaflutole. Pestic. Bioch. Physiol., v. 62, p. 125-134, 1998. 